\title{
Original Research \\ The association of vascular endothelial growth factor related SNPs and circulating iron levels might depend on body mass index
}

\author{
Pia Chedid ${ }^{1,2, \dagger}$, Ali Salami ${ }^{1,3, \dagger}$, Mariam Ibrahim $^{4}$, Sophie Visvikis-Siest ${ }^{1}$, Said El Shamieh ${ }^{5, *}$ \\ ${ }^{1}$ Université de Lorraine, IGE-PCV, F-54000 Nancy, France \\ ${ }^{2}$ Department of Medical Laboratory Sciences, Faculty of Health Sciences, University of Balamand, 1100-2807 Beirut, Lebanon \\ ${ }^{3}$ Department of Mathematics, Faculty of Sciences, Lebanese University, 1700 Nabatieh, Lebanon \\ ${ }^{4}$ Rammal Hassan Rammal Research Laboratory, PhyToxE Research Group, Faculty of Sciences (V), Lebanese University, 1700 Nabatieh, Lebanon \\ ${ }^{5}$ Department of Medical Laboratory Technology, Faculty of Health Sciences, Beirut Arab University, 115020 Beirut, Lebanon \\ *Correspondence: s.elshamieh@bau.edu.lb (Said El Shamieh) \\ $\dagger$ These authors contributed equally. \\ Academic Editor: Graham Pawelec \\ Submitted: 26 May 2021 Revised: 9 August 2021 Accepted: 24 August 2021 Published: 18 January 2022
}

\begin{abstract}
Background and objectives: Vascular Endothelial Growth Factor (VEGF) is an essential regulator of vascular biology. In addition to the well-established role in angiogenesis, circulating VEGF levels were found elevated in severely anemic patients, pointing out that anemia might affect the progression of angiogenesis in malignant and benign diseases through the alteration of VEGF levels. Ten single nucleotide polymorphisms (SNPs) in VEGFA and other loci were shown to explain more than $50 \%$ of its circulating levels. This study investigated the association of those ten VEGF-related SNPs with serum iron levels in a general Lebanese population free of chronic diseases $(\mathrm{N}=460)$. Result: We found that the rs 10738760 and the body mass index (BMI) were associated with decreased Iron levels ( $p=0.002$, and $p<0.001$, respectively). When taken together, both variables, rs 10738760 and BMI, interacted to reduce iron levels $(p$ $<0.001)$. According to obesity status, the stratification revealed that the effect of rs 10738760 was more pronounced in obese than nonobese individuals $(p=0.025)$. Conclusion: The intergenic SNP rs 10738760 is associated with circulating iron levels, and this association depends on BMI status. Although of interest, these results need replication in larger populations from different ancestries.
\end{abstract}

Keywords: Vascular endothelial growth factor; SNPs; Iron levels; Body mass index

\section{Introduction}

Low serum iron levels have significant pathologic consequences, including but not limited to anemia [1]. Several predisposing factors were reported, such as female sex and microbial infections [2,3]. Some previous studies have suggested that increased body mass index (BMI) might be a risk factor for low serum iron $[4,5]$. Population-based studies showed that low serum iron levels are associated with an increased risk of CVD, supporting the possibility that there is an inverse association between serum iron levels and risk of mortality [6]. Going into the same direction, evidence from cross-sectional case-control studies revealed that serum iron was significantly lower in patients with myocardial infarction than in controls, suggesting that low stored iron levels are a risk factor for premature CVD [7]. The first study addressing iron status in children and adolescents in Lebanon revealed that $14.2 \%$ of the Lebanese pediatrics population had iron insufficiency with females having greater incidence than boys [8]. Moreover, a cross sectional study that was done on Lebanese women who visited governmental healthcare facilities showed that the percentages of women suffering from anemia and iron deficiency are respectively about $16 \%$ and $27 \%$ of the study population (totally $7.7 \%$ had iron deficiency anemia) [9].
A new updated study showed that approximately $60 \%$ of the Lebanese Women have dietary iron intake deficiency, with low-income women being the most impacted [10].

Vascular endothelial growth factor (VEGF, also referred to as VEGFA) is an essential regulator of vascular biology [11]. Specifically, it stimulates angiogenesis in a wide range of processes (both normal and pathological) [11]. In addition to the well-established role in angiogenesis, levels of circulating VEGF were found elevated in severely anemic patients [12], pointing out that anemia might affect the progression of angiogenesis in malignant and benign diseases via the alteration of VEGF levels $[12,13]$.

The heritability of its circulating levels is high and estimated to be between $60 \%$ and $80 \%$ [14]. In a genomewide association study (GWAS), Debette et al. [15] identified four single nucleotide polymorphisms (SNPs); rs6921438 and rs4416670 in LOC100132354-C6orf223, rs6993770 in ZFPM2, and rs 10738760 in VLDLR-KCNV2 that explained up to $50 \%$ of the heritability of VEGFA circulating levels. Specifically, two SNPs explained a significant proportion of the heritability of circulating VEGF levels: rs6921438 and $\mathrm{rs} 10738760(41.2 \%$ and $5.0 \%$ respectively) [15]. A more recent GWAS study conducted on a total of 16,112 samples of European individuals identified six 
additional genetic variants at the novel $(M E F 2 C, J M J D 1 C$, ZFPM1, and ZADH2) and known loci (LOC100132354, C6orf223, ZFPM2, and KCNV2) [16]. VEGF SNPs; rs6921438 was associated with decreased HDL-C and increased LDL-C levels in supposedly healthy European individuals [17], rs6993770, and rs10738760 were shown to have positive relationships with metabolic syndrome and hypercholesterolemia in two Middle Eastern populations $[18,19]$. Therefore, this study investigated the association of those ten SNPs and BMI with serum iron levels in a general Lebanese population (LGP) composed of 460 individuals with no chronic disease.

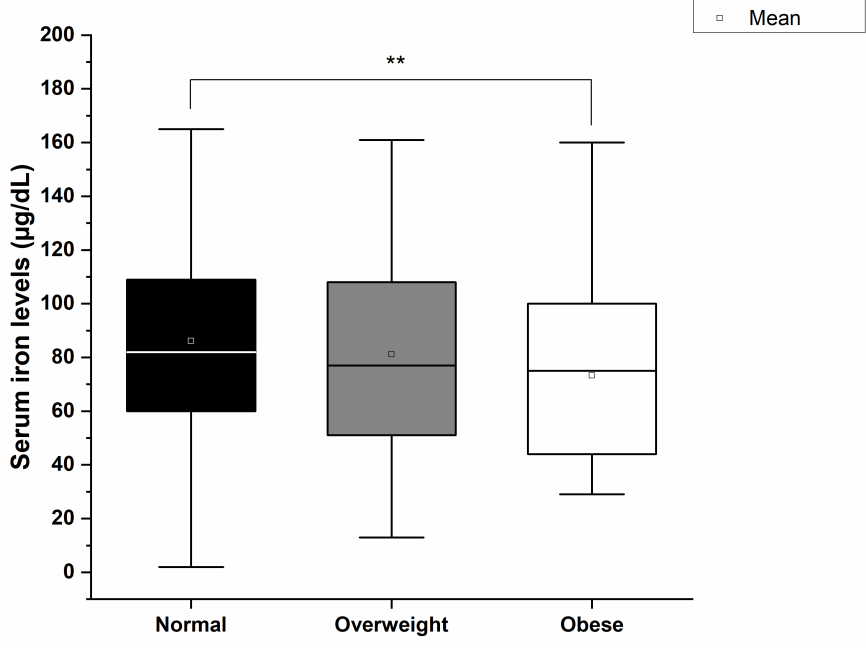

Fig. 1. Levels of serum iron according to BMI categories. ** significant difference between Normal and Obese groups ( $p$ $=0.003)$

\section{Material and methods}

\subsection{Clinical, biological, and genetic data}

Socio-demographic were assessed using a questionnaire. Normal weight was defined as body mass index (BMI) $<25 \mathrm{Kg} / \mathrm{m}^{2}$, overweight and obese were defined as individuals having a BMI between $25-29.99 \mathrm{Kg} / \mathrm{m}^{2}$ and $\geq 30 \mathrm{Kg} / \mathrm{m}^{2}$, respectively. Iron levels were considered normal if $65-176 \mu \mathrm{g} / \mathrm{dL}$ and $50-170 \mu \mathrm{g} / \mathrm{dL}$ in males and females, respectively. Serum iron levels were analyzed using Roche reagents on the Roche/Hitachi Modular P instrument (Roche Diagnostics, Indianapolis, IN). Total genomic DNA was extracted from peripheral blood samples (QIAamp DNA blood mini kit, Qiagen, Hilden, Germany). The ten SNPs of VEGF were genotyped using the KASP technique (FRET-based) described previously [19,20].

\subsection{Statistical analysis}

All analyses were performed using SPSS statistical software version 24.0 (SPSS, Inc.; Chicago, IL, USA). Nor- mality was tested using Kolmogorov-Smirnov test. Kruskal Wallis test was used to study if there is a significant difference in the mean of the serum iron levels between BMI categories (Normal, Overweight, and Obese). Then a post hoc analysis was performed using Wilcoxon rank-sum test to confirm where the differences occurred between which groups. A chi-square test was performed to test the HardyWeinberg equilibrium and to evaluate whether a significant difference was present between the categorical variables. All genetic analyses were performed using an additive model.

We used the minor allele as the reference allele in all our analysis. Linear regression models adjusted for age, gender, and BMI were used to assess the effect of each SNP on serum iron levels. Only the significant SNPs were shown. The significance level was set at $p \leq 0.005$ due to the multiple testing performed on 10 VEGF SNPs. The contribution of "SNP $\times$ BMI" interaction was also assessed within our model after adjustment for age and gender; the significance level was set at $p \leq 0.05$.

A multivariate logistic regression model was used to study the association between the SNPs and serum iron status used while correcting for age, gender, and BMI. Only the significant SNPs were shown. The significance level was set at $p \leq 0.005$ because of multiple testing (10 SNPs).

\section{Results}

All the participants' characteristics were presented in Tables 1,2, respectively. About $50 \%$ of our participants are of normal BMI, approximately $25 \%$ are overweight, and about $20 \%$ are obese. All studied SNPs agreed with the Hardy-Weinberg equilibrium. The levels of serum iron according to BMI categories are shown in Fig. 1. There was a statistically significant difference in the mean of the serum iron levels between BMI categories as determined by Kruskal Wallis test ( $p=0.009$, Fig. 1). A post hoc analysis using Wilcoxon rank-sum test revealed that the serum iron level was statistically significantly higher in the normal group $(86.15 \pm 36.05(\mu \mathrm{g} / \mathrm{dL}), p=0.003)$ compared to obese group $(73.27 \pm 30.60(\mu \mathrm{g} / \mathrm{dL}))$. The minor allele frequencies of VEGF SNPs were shown in Table 2.

The association of the 10 VEGF-related SNPs and BMI with serum iron levels revealed three significant variations that are shown in Table 3. The multivariate linear regression analysis reveals that rs10738760 and BMI are both associated significantly with decreased serum iron level ( $p=0.002$ and $p<0.001$ respectively). However, rs2639990 and rs6921438 are not associated significantly with decreased serum iron levels ( $p=0.027$ and $p=0.088$, respectively). Age and gender showed a significant association with serum iron status $(p<0.001)$.

On the other hand, the interactions between rs10738760, rs2639990 and rs6921438 with BMI decreased significantly the iron levels $(p<0.001, p=$ $0.001, p=0.001$, respectively) (Table 3). Furthermore, 
Table 1. Characteristics of the study participants.

\begin{tabular}{lcc}
\hline & \multicolumn{2}{c}{ Lebanese general population $(\mathrm{N}=460)$} \\
\cline { 2 - 3 } & Mean & $\mathrm{SD}^{2}$ \\
\hline Age (years) & 40.6 & 14.16 \\
Gender (female \%) & 63.5 & \\
Body mass index $\left(\mathrm{kg} / \mathrm{m}^{2}\right)$ & 25.71 & 4.98 \\
Normal weight $(<25)$ & $253(55.0 \%)$ & \\
Overweight $(25-29.9)$ & $114(24.8 \%)$ & \\
Obesity $(\geq 30)$ & $93(20.2 \%)$ & \\
Smoking $(\%)$ & 26.5 & \\
Alcohol consumption $(\%)$ & 35.2 & \\
Marriage $(\%)$ & 69.8 \\
Serum iron level $(\mu \mathrm{g} / \mathrm{dL})$ & 82.3 \\
Low serum levels & $110(23.9 \%)$ \\
Normal serum levels & $350(76.1 \%)$ & 35.75 \\
\hline
\end{tabular}

${ }^{1}$ Mean value for continuous variables and a percentage for categorical variables.

${ }^{2} \mathrm{SD}$, standard deviation (only for continuous variables).

Table 2. The minor allele frequencies of $V E G F$ single nucleotide polymorphisms.

\begin{tabular}{lc}
\hline MAF of VEGF SNPs & \\
\hline rs10738760A $>$ G & 0.46 \\
rs6993770A $>$ T & 0.34 \\
rs6921438G $>$ A & 0.34 \\
rs4416670C $>$ T & 0.5 \\
rs2639990A $>$ G & 0.11 \\
rs114694170T $>C$ & 0.02 \\
rs4782371T $>$ G & 0.44 \\
rs10761741G $>$ T & 0.41 \\
rs7043199T $>$ A & 0.21 \\
rs34528081T - & 0.38 \\
\hline
\end{tabular}

MAF, minor allele frequency; SNPs, single nucleotide polymorphisms.

a multivariate linear regression analysis of serum iron levels in obese and non-obese participants was performed, resulting in the finding that the rs 1078760 effect was more pronounced in obese than non-obese individuals ( $p=0.025$ vs. $p=0.039$, Table 4 ).

Additionally, multiple logistic regression analyses of risk factors with obesity were applied, as shown in Table 5. The obtained results showed that VEGF-related SNP rs 10738760 was significantly associated with decreased serum iron level $(p=0.003)$. Besides, obese status was also associated significantly with decreased serum iron levels $(p$ $<0.001)$. Hence, rs10738760 and obesity are highly associated with an increased risk of low iron. Age was found to increase the risk of low serum iron levels.
Table 3. Multivariate linear regression analysis with serum iron level.

\begin{tabular}{lcccc}
\hline & \multicolumn{4}{c}{ Serum iron level $(\mu \mathrm{g} / \mathrm{dL})$} \\
\cline { 2 - 5 } & $\mathrm{B}$ & $\mathrm{SE}$ & $\beta$ & $p$ \\
\hline Age & 0.60 & 0.12 & 0.24 & $<0.001$ \\
Gender & -21.17 & 3.54 & -0.29 & $<0.001$ \\
BMI & -1.87 & 0.34 & -0.26 & $<0.001$ \\
rs10738760 & -6.91 & 2.26 & -0.14 & 0.002 \\
rs2639990 & -8.23 & 3.70 & -0.10 & 0.027 \\
rs6921438 & -3.97 & 2.32 & -0.08 & 0.088 \\
rs10738760 $\times$ BMI* $^{*}$ & -0.32 & 0.08 & -0.19 & $<0.001$ \\
rs2639990 $\times$ BMI* $^{*}$ & -0.47 & 0.13 & -0.16 & 0.001 \\
rs6921438 $\times$ BMI* $^{*}$ & -0.27 & 0.08 & -0.15 & 0.001 \\
\hline
\end{tabular}

*BMI interaction with genotypes was studied in a separate model adjusted for age and gender. B: Unstandardized coefficients. SE, Standard Error. $\beta$ : Standardized coefficients.

Table 4. Multivariate linear regression analysis with serum iron level according to obesity status.

\begin{tabular}{lcccccccc}
\hline & \multicolumn{4}{c}{ Obese } & \multicolumn{4}{c}{ Non-Obese } \\
\cline { 2 - 9 } & B & SE & $\beta$ & $p$ & B & SE & $\beta$ & $p$ \\
\hline Age & 0.48 & 0.18 & 0.19 & 0.008 & 0.57 & 0.13 & 0.22 & $<0.001$ \\
Gender & -42.33 & 4.43 & -0.68 & $<0.001$ & -12.07 & 4.12 & -0.15 & 0.004 \\
rs10738760 & -6.99 & 3.07 & -0.16 & 0.025 & -5.41 & 2.61 & -0.11 & 0.039 \\
rs2639990 & -9.53 & 7.01 & -0.09 & 0.178 & -6.54 & 4.16 & -0.08 & 0.117 \\
rs6921438 & -5.53 & 3.20 & -0.12 & 0.087 & -1.68 & 2.71 & -0.03 & 0.536 \\
\hline
\end{tabular}

B: Unstandardized coefficients. $\beta$ : Standardized coefficients.

SE, Standard Error.

\section{Discussion}

All results indicate that the VEGF-related SNP rs10738760 is associated with circulating iron levels, and this association depends on BMI status. The mean serum iron level decreases with increased BMI. Both rs 10738760 and BMI are associated with lowered serum iron levels. Together, rs10738760 and BMI are found to interact to decrease iron levels. Besides, the impact of rs 10738760 is more notable in obese individuals than non-obese ones.

Different studies have reported the negative impact of obesity on iron levels across all ages [21]. According to the Third National Health and Nutrition Examination Survey (NHANES III), the risk of suffering from iron deficiency is two times higher in obese youngsters than others with average weight, and the same results have been obtained in adults [22]. The mechanism that explains the linkage between obesity status and iron levels remains unclear [22]. However, findings suggest that low iron-diet consumption, diminished intestinal absorption of iron, and increased iron demand due to the larger blood volume may be the underlying contributors to iron deficiency in obese people [21,22]. 
Table 5. Multiple logistic regression analysis with serum iron status.

\begin{tabular}{|c|c|c|c|}
\hline \multicolumn{4}{|c|}{ Serum iron status } \\
\hline & & OR (95\% C.I.) & $p$ \\
\hline \multirow[t]{2}{*}{ Age } & $<40$ & 1 & \\
\hline & $\geq 40$ & $0.55(0.33-0.92)$ & 0.021 \\
\hline \multirow[t]{2}{*}{ Gender } & Male & 1 & \\
\hline & Female & $0.83(0.49-1.40)$ & 0.493 \\
\hline \multirow[t]{3}{*}{ BMI } & $<25$ & 1 & \\
\hline & $25-29.9$ & $0.49(0.27-0.88)$ & 0.017 \\
\hline & $\geq 30$ & $0.30(0.16-0.57)$ & $<0.001$ \\
\hline \multirow[t]{3}{*}{ rs 10738760} & AA & 1 & \\
\hline & GA & $0.94(0.52-1.69)$ & 0.824 \\
\hline & GG & $0.36(0.19-0.71)$ & 0.003 \\
\hline
\end{tabular}

OR, Odds Ratio; C.I., Confidence Interval. Normal serum levels were considered as the reference group.

Another hypothesis is chronic inflammation [21,22]. Obesity is linked to persistent chronic inflammation in response to high adiposity that has also been linked with low serum iron levels [21]. Yanoff et al. [23] has reported the existence of elevated levels of C-reactive protein (CRP) in obese populations. Additionally, Zimmermann et al. [24] have revealed that the increased adiposity is linked with decreased iron absorption. Chronic inflammation is associated with the secretion of pro-inflammatory cytokines like interleukin-6 (IL-6) and tumor necrosis factor- $\alpha$ (TNF- $\alpha$ ) that may trigger liver and adipose tissue to release hepcidin which is likely to decrease iron absorption [22]. Hence, iron sequestration by the inflammatory mediated pathway may be one of the potential origins of the low iron level in obese subjects [21]. Hence, serum iron concentration decreases as BMI increases [25].

VEGF is involved in the pathogenesis of obesity [26]. Being an endocrine organ, the fat tissue releases significant amounts of VEGF, inducing angiogenesis [26,27]. Plasma VEGF was detected in significantly higher amounts in obese people than normal weight and lean subjects [26]. Miyazawa-Hoshimoto et al. [28] have reported that individuals with high BMI and visceral fat aggregation tend to have higher serum VEGF. It was suggested that the elevated level of serum VEGF coupled with increased adiposity might affect the vascular endothelial function via triggering the endothelial cells to migrate and proliferate, increasing the permeability of blood vessels, and controlling thrombogenicity [28].

Furthermore, the transcription factor hypoxiainducible factor- $1 \alpha$ (HIF-1 $\alpha$ ) activity was found to be elevated in adipose tissue that may underlie chronic inflammation in obese subjects [29]. HIF-1 $\alpha$ activity in obese individuals is mainly induced by hypoxia, insulin, and adipogenesis [29]. HIF-1 $\alpha$ acts as a critical transcriptional activator for the VEGF gene [29]. Hence, adipose tissue is thought to exhibit a significant level of VEGF in response to the HIF- $1 \alpha$ pathway, which provides a molecular mechanism for the high ability of adipose tissue in inducing angiogenesis [29].

On the other hand, a large body of work has shown that VEGF formation can be driven by iron deficiency [30]. Interestingly, it was found that mice fed with diets lacking iron exhibited elevated levels of HIF- $1 \alpha$ and VEGF in comparison to mice fed with diets rich with iron [30]. Iron deficiency may also increase the production of VEGF through activation of the HIF-1 $\alpha$ pathway [30]. Iron deficiency has been proved to be a substantial factor that triggers increased VEGF levels [31].

Supporting this study, females are found to be at an elevated risk of developing iron deficiency anemia due to menstrual iron losses [32]. Besides, pregnant and lactating women are considered to be at higher risk of encountering iron deficiency [32]. Moreover, iron deficiency anemia is common in the elderly population [33]. This may be due to poor dietary intake, diminished efficiency of iron absorption, bacterial infections, gastrointestinal bleeding, some medications, or chronic complications [33].

This is the first study that reveals the association of VEGF-related polymorphisms with serum iron status and the potential dependence of this relation on BMI to the best of our knowledge. This study demonstrates three limitations; (1) female subjects were more abundant than males with a ratio of $2: 1$, demanding adjustment for gender to eliminate any confounding effect, (2) the absence of replication in larger populations, (3) several factors that may affect both iron status and BMI, like socioeconomic factors and physical activity were missing. (4) The lack of VEGF levels measurement in the plasma. (5) Despite our report showing that VEGF-related SNP rs1073760 is associated with circulating iron levels and depends on BMI, we cannot exclude that this association might result from the link between rs1073760 and BMI and not from direct association. This is because previous studies have reported that SNP rs1073760 were shown to have positive relationship with metabolic syndrome $[18,19]$ and serum iron concentration decrease as BMI increases [25].

In conclusion, the intergenic VEGF-related SNP rs 10738760 is associated with circulating iron levels, and this association depends on BMI. These findings need to be validated in larger populations and settings. Further investigations are needed to elucidate the molecular mechanism of VEGF polymorphism and BMI interactions in decreasing serum iron levels. Understanding this relationship may allow the development of dietary or pharmacologic therapies that could reduce the risk of developing an iron deficiency in obese individuals. 


\section{Author contributions}

PC, AS and SES conceived and designed the experiments; MI and PC performed the experiments; AS analyzed the data; SVS and SES contributed reagents and materials, PC, AS and MI wrote the first draft, SES revised the manuscript.

\section{Ethics approval and consent to participate}

All the recruitment and genetic procedures were done following the latest version of the Declaration of Helsinki for Ethical Principles for Medical Research Involving $\mathrm{Hu}$ man Subjects. The Institutional Review Board of the Beirut Arab University approved the study (2019-H-0091-HS-R0360). Every participant gave informed consent before participation.

\section{Acknowledgment}

We thank the participants and their families for making part of this study.

\section{Funding}

This research received no external funding.

\section{Conflict of interest}

The authors declare no conflict of interest.

\section{References}

[1] Stoltzfus RJ. Iron Deficiency: Global Prevalence and Consequences. Food and Nutrition Bulletin. 2003; 24: S99-S103.

[2] Ramzi M, Haghpanah S, Malekmakan L, Cohan N, Baseri A, Alamdari A, et al. Anemia and iron deficiency in adolescent school girls in kavar urban area, southern iran. Iranian Red Crescent Medical Journal. 2011; 13: 128-133.

[3] Yang Y, Sheu B, Lee S, Yang H, Wu J. Children of Helicobacter pylori-infected dyspeptic mothers are predisposed to H. pylori acquisition with subsequent iron deficiency and growth retardation. Helicobacter. 2005; 10: 249-255.

[4] Cepeda-Lopez AC, Osendarp SJ, Melse-Boonstra A, Aeberli I, Gonzalez-Salazar F, Feskens E, et al. Sharply higher rates of iron deficiency in obese Mexican women and children are predicted by obesity-related inflammation rather than by differences in dietary iron intake. The American Journal of Clinical Nutrition. 2011; 93: 975-983.

[5] Tussing-Humphreys LM, Liang H, Nemeth E, Freels S, Braunschweig CA. Excess Adiposity, Inflammation, and IronDeficiency in Female Adolescents. Journal of the American Dietetic Association. 2009; 109: 297-302.

[6] Corti MC, Guralnik JM, Salive ME, Ferrucci L, Pahor M, Wallace RB, et al. Serum iron level, coronary artery disease, and allcause mortality in older men and women. The American Journal of Cardiology. 1997; 79: 120-127.

[7] Regnström J, Tornvall P, Kallner A, Nilsson J, Hamsten A. Stored iron levels and myocardial infarction at young age. Atherosclerosis. 1994; 106: 123-125.

[8] El Khoury R, Sleilaty G, Gannagé-Yared M. Prevalence of Iron deficiency in Lebanese schoolchildren. European Journal of Clinical Nutrition. 2020; 74: 1157-1163.

[9] Al Khatib L, Obeid O, Sibai A, Batal M, Adra N, Hwalla N. Folate deficiency is associated with nutritional anaemia in
Lebanese women of childbearing age. Public Health Nutrition. 2006; 9: 921-927.

[10] Doumani N, Maalouly J, Bou-Maroun E, Sok N, Cayot P, Tueni $M$. Iron intake among Lebanese women: sociodemographic factors, iron-rich dietary patterns, and preparation of hummus, a Mediterranean dish. Food \& Nutrition Research. 2021; 65.

[11] Ferrara N, Gerber H, LeCouter J. The biology of VEGF and its receptors. Nature Medicine. 2003; 9: 669-676.

[12] Dunst J, Becker A, Lautenschläger C, Markau S, Becker H, Fischer K, et al. Anemia and elevated systemic levels of vascular endothelial growth factor (VEGF). Strahlentherapie Und Onkologie. 2002; 178: 436-441.

[13] Dunst J, Pigorsch S, Hänsgen G, Hintner I, Lautenschläger C, Becker A. Low hemoglobin is associated with increased serum levels of vascular endothelial growth factor (VEGF) in cancer patients. Does anemia stimulate angiogenesis? Strahlentherapie Und Onkologie. 1999; 175: 93-96.

[14] Berrahmoune H, Herbeth B, Lamont JV, Masson C, Fitzgerald PS, Visvikis-Siest S. Heritability for plasma VEGF concentration in the Stanislas family study. Annals of Human Genetics. 2007; 71: 54-63.

[15] Debette S, Visvikis-Siest S, Chen M, Ndiaye N, Song C, Destefano A, et al. Identification of cis- and trans-acting genetic variants explaining up to half the variation in circulating vascular endothelial growth factor levels. Circulation Research. 2011; 109: 554-563.

[16] Choi SH, Ruggiero D, Sorice R, Song C, Nutile T, Vernon Smith A, et al. Six Novel Loci Associated with Circulating VEGF Levels Identified by a Meta-analysis of Genome-Wide Association Studies. PLoS Genetics. 2016; 12: e1005874.

[17] Stathopoulou MG, Bonnefond A, Ndiaye NC, Azimi-Nezhad M, El Shamieh S, Saleh A, et al. A common variant highly associated with plasma VEGFA levels also contributes to the variation of both LDL-C and HDL-C. Journal of Lipid Research. 2013; 54: 535-541.

[18] Azimi-Nezhad M, Mirhafez SR, Stathopoulou MG, Murray H, Ndiaye NC, Bahrami A, et al. The Relationship between Vascular Endothelial Growth Factor Cis- and Trans-Acting Genetic Variants and Metabolic Syndrome. The American Journal of the Medical Sciences. 2018; 355: 559-565.

[19] Salami A, El Shamieh S. Association between SNPs of Circulating Vascular Endothelial Growth Factor Levels, Hypercholesterolemia and Metabolic Syndrome. Medicina. 2019. 55: 464.

[20] Assaad S, Costanian C, Jaffal L, Tannous F, Stathopoulou MG, El Shamieh S. Association of TLR4 Polymorphisms, Expression, and Vitamin D with Helicobacter pylori Infection. Journal of Personalized Medicine. 2019; 9: 2.

[21] Ghadiri-Anari A, Nazemian N, Vahedian-Ardakani H. Association of body mass index with hemoglobin concentration and iron parameters in Iranian population. ISRN Hematology. 2014; 2014: 525312 .

[22] McClung JP, Karl JP. Iron deficiency and obesity: the contribution of inflammation and diminished iron absorption. Nutrition Reviews. 2009; 67: 100-104.

[23] Yanoff LB, Menzie CM, Denkinger B, Sebring NG, McHugh $\mathrm{T}$, Remaley AT, et al. Inflammation and iron deficiency in the hypoferremia of obesity. International Journal of Obesity. 2007; 31: $1412-1419$.

[24] Zimmermann MB, Zeder C, Muthayya S, Winichagoon P, Chaouki N, Aeberli I, et al. Adiposity in women and children from transition countries predicts decreased iron absorption, iron deficiency and a reduced response to iron fortification. International Journal of Obesity. 2008; 32: 1098-1104.

[25] Aigner E, Feldman A, Datz C. Obesity as an emerging risk factor for iron deficiency. Nutrients. 2014; 6: 3587-3600.

[26] Loebig M, Klement J, Schmoller A, Betz S, Heuck N, Schweiger $\mathrm{U}$, et al. Evidence for a relationship between VEGF and BMI 
independent of insulin sensitivity by glucose clamp procedure in a homogenous group healthy young men. PLoS ONE. 2010; 5: e12610.

[27] Wieczór R, Wieczór AM, Gadomska G, Stankowska K, Fabisiak J, Suppan K, et al. Overweight and obesity versus concentrations of VEGF-a, sVEGFR-1, and sVEGFR-2 in plasma of patients with lower limb chronic ischemia. Journal of Zhejiang University. Science B. 2016; 17: 842-849.

[28] Miyazawa-Hoshimoto S, Takahashi K, Bujo H, Hashimoto N, Saito Y. Elevated serum vascular endothelial growth factor is associated with visceral fat accumulation in human obese subjects. Diabetologia. 2003; 46: 1483-1488.

[29] He Q, Gao Z, Yin J, Zhang J, Yun Z, Ye J. Regulation of HIF1alpha activity in adipose tissue by obesity-associated factors: adipogenesis, insulin, and hypoxia. American Journal of Physiology. Endocrinology and Metabolism. 2011; 300: E877-E885.

[30] Jian J, Yang Q, Dai J, Eckard J, Axelrod D, Axelrod D, et al.
Effects of iron deficiency and iron overload on angiogenesis and oxidative stress-a potential dual role for iron in breast cancer. Free Radical Biology \& Medicine. 2011; 50: 841-847.

[31] Hoseini Z, Azimi-Nezhad M, Ghayour-Mobarhan M, Avan A, Eslami S, Nematy M, et al. VEGF gene polymorphism interactions with dietary trace elements intake in determining the risk of metabolic syndrome. Journal of Cellular Biochemistry. 2018. (in press)

[32] Levi M, Simonetti M, Marconi E, Brignoli O, Cancian M, Masotti A, et al. Gender differences in determinants of irondeficiency anemia: a population-based study conducted in four European countries. Annals of Hematology. 2019; 98: 15731582.

[33] Fairweather-Tait SJ, Wawer AA, Gillings R, Jennings A, Myint PK. Iron status in the elderly. Mechanisms of Ageing and Development. 2014; 136-137: 22-28. 